\title{
Experimental evidence for two different dynamical regimes in liquid rubidium
}

\author{
Franz Demmel ${ }^{1, *}$ and Christoph Morkel $^{2, * *}$ \\ ${ }^{1}$ ISIS Facility, Rutherford Appleton Laboratory, Didcot, OX11 0QX, UK \\ ${ }^{2}$ Physikdepartment E21, TU München, 85748 Garching, Germany
}

\begin{abstract}
We present evidence for changes in the dynamics of liquid rubidium with rising temperature. The thermal expansion of this liquid alkali metal shows a changing derivative with temperature in a temperature range of about 400-500 K. With neutron scattering the amplitude at the structure factor maximum demonstrates a changing slope with increasing temperature. A derived averaged structural relaxation time can be understood that an additional relaxation process sets in upon cooling. The deduced generalized viscosity and high frequency shear modulus indicate a change in dynamics in the same temperature range. All these findings point to a change in dynamics of the equilibrium liquid metal state with a dynamical crossover from a viscous to a fluid-like liquid metal well above the melting point.
\end{abstract}

\section{Introduction}

During the past decades the collective dynamics of liquid metals has been intensively investigated near the melting point, e.g. for an earlier and a more recent review on the dynamics of liquid metals see $[1,2]$. Liquid alkali metals have been studied in particular, because they show distinct inelastic excitations [3-7]. One common finding was the upward bending of the frequency dispersion above the values expected from hydrodynamics, which is called positive dispersion. This enhancement in frequency can be described as a viscoelastic reaction of the fluid. At low frequencies the relaxations in the liquid can follow the density fluctuations and the sound waves propagate with the adiabatic sound velocity. At high frequencies the liquid response becomes solid-like with a transition to a high frequency sound velocity. A formal description can be obtained within the so-called viscoelastic model, which incorporates the two different dynamic regimes separated by a relaxation time $\tau$, referred to as Maxwell's relaxation time.

However, the temperature dependence of the collective dynamics was rarely studied. One of the few studies explored the changes of the collective excitations with temperature in liquid sodium up to the boiling point in four coarse temperature steps [6]. Further investigations on changes in the dynamics with rising temperature up to the boiling point and beyond focused on the self-diffusion of liquid alkali metals and the underlying changes in the dynamics with decreasing density [8].

\footnotetext{
*e-mail: franz.demmel@stfc.ac.uk

**e-mail: christoph.morkel@frm2.tum.de
} 
Less into the focus of research have been the changes in dynamics between the melting point and a few hundred degree above it, when the density changes only about $10 \%$. However, recent studies demonstrated distinct changes in the rubidium dynamics on atomic length scales with increasing temperature from the melting point $T_{\text {melt }}=312.65 \mathrm{~K}$ up to about twice the melting temperature [9-12]. The alkali metal rubidium has been studied for several decades and a lot of data, macroscopic and microscopic, are available over a wide temperature range from experiment and simulation (e.g.[13-17]). Therefore, rubidium represents an ideal test case to investigate changes in liquid metal dynamics with rising temperature in detail. With neutrons there is a long tradition of studying liquid rubidium due to its favorable scattering properties, namely the negligible incoherent cross section, low absorption and a reasonable large coherent cross section. Most of the experiments were dedicated to investigate inelastic properties of liquid rubidium $[3,18]$. Towards the end point of the liquid gas phase boundary, the critical point, measurements of the structure and collective dynamics have been performed [19-21]. More specifically, a change in the current spectra has been related to the onset of dimerization in the proximity of the metal-nonmetal transition. However, most of the previous microscopic experimental or simulation work on liquid rubidium were concerned with quite coarse steps in temperature away from the melting point.

Here we assess the obtained data on liquid rubidium in a novel way, extract average structural relaxation times from the amplitude at zero energy transfer and derive the elastic shear modulus from the analysis of the acoustic-type excitations with rising temperature. We will relate the observed changes to the temperature dependence of a macroscopic parameter, the thermal expansion. The comparison of the collected data represents a coherent picture about the changes in dynamics between melting point and twice $T_{\text {melt }}$.

\section{Experimental details}

The coherent dynamic structure factor $S(Q, \omega)$ of liquid rubidium has been measured by neutron scattering. Rubidium is a nearly coherent scatterer with an incoherent contribution of less than 0.04 barn compared to the coherent cross section of 6.24 barn [22]. Rubidium (99.9 $\%$ purity) was filled in a cylindrical aluminium can with $16 \mathrm{~mm}$ diameter ( $9 \%$ scatterer) inside a glove box. The cell has been electron beam welded to keep the sample vacuum tight. The structure factor maximum of liquid rubidium occurs at $\mathrm{Q}_{0}=1.52 \AA^{-1}$. The melting temperature of rubidium is $T=312.65 \mathrm{~K}$. Three experiments with differing energy resolutions have been performed to follow the changes in dynamics at the structure factor maximum. A first series of experiments has been performed at the three axis spectrometer with multianalyzer-detector unit of the Forschungsreaktor München FRM [23]. Constant-Q scans at thirteen temperatures have been measured with an energy resolution of $F W H M=1.3 \mathrm{meV}$. A second series of experiments has been performed at the thermal three axis spectrometer IN3 at the Institute Laue Langevin, Grenoble. At a final energy of $14.66 \mathrm{meV}$ an energy resolution of $0.8 \mathrm{meV}$ was obtained. A third series of experiments was undertaken at the IRIS-spectrometer of the ISIS Facility. A configuration with an end energy of $7.38 \mathrm{meV}$ was chosen, which provided an excellent energy resolution of $0.055 \mathrm{meV}$ (FWHM). More experimental details can be found in $[9,12,23]$. Furthermore, inelastic experiments to follow the collective excitations at $320 \mathrm{~K}, 420 \mathrm{~K}, 500 \mathrm{~K}$ and $600 \mathrm{~K}$ have been performed and analysed [11]. More details about the extensive data analysis and comparison with MD-simulations can be found there. 


\section{Results and Discussion}

At first we consider a macroscopic quantity, the volumetric thermal expansion coefficient: $\alpha=\frac{1}{V}\left(\frac{d V}{d T}\right)_{P}$. In Fig 1 we plot the thermal expansion and the mass density of liquid rubidium obtained through macroscopic measurements [24]. Apparently the slope of the thermal expansion changes with rising temperature in a temperature range from $400 \mathrm{~K}$ to $500 \mathrm{~K}$. The inset shows the measured mass density, which already indicates this change with a changing slope. Within fluctuation theory the thermal expansion is related to correlated fluctuations

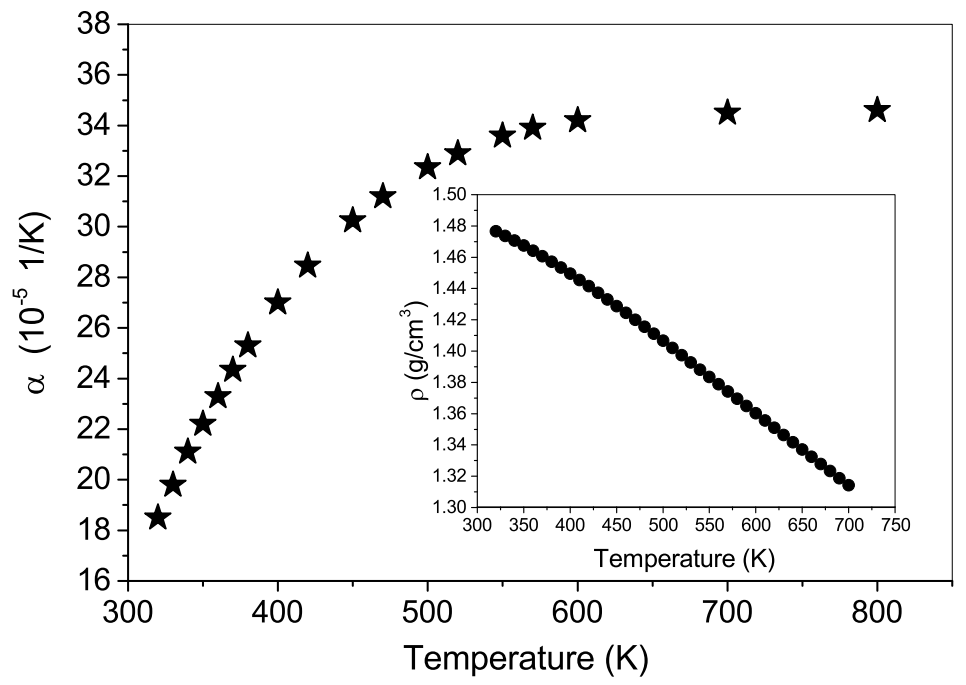

Figure 1. The thermal expansion coefficient of liquid rubidium is presented over a large range of temperatures [24]. The inset shows the change of mass density with increasing temperature.

of volume and entropy [25]. It appears that the correlations between volume and entropy fluctuations are changing much more in a temperature range up to about $400-500 \mathrm{~K} \mathrm{com}-$ pared to the higher temperatures. That change in fluctuation correlations hints to a changing microscopic dynamics within that temperature range.

Now we want to relate these fluctuations on a macroscopic scale with density fluctuations on an atomic length scale through coherent neutron scattering. Coherent neutron scattering provides insight into the collective movements of the particles. In the hydrodynamic limit the scattering function is given by a combination of three Lorentzians, neglecting a small asymmetry term [26]. For larger momentum transfers or when length scales of atomic diameters are probed the quasielastic line shows a narrowing around the structure factor maximum, known as deGennes narrowing [27]. In the time domain the intermediate scattering function for density fluctuations $F(Q, t)$ demonstrates a slowing down at the structure factor maximum. In a simple picture it costs time for a density fluctuation to relax on a next-neighbor length scale due to a necessary rearrangement of the surrounding particles. That necessary time cost appears in the frequency domain as a narrowing of the quasielastic line. On this length scale the most direct access to the evolution of structural relaxation is provided.

We analysed the amplitude at the structure factor maximum $S\left(Q_{0}, \omega=0\right)$ from three different measurements on liquid rubidium, all performed with different energy resolutions. Fig. 2 depicts the derived amplitudes $S\left(Q_{0}, \omega=0\right)$. The inset illustrates the change of the spectral amplitude with rising temperature. From $320 \mathrm{~K}$ to $400 \mathrm{~K}$ the amplitude changes 
about twice as much as between $400 \mathrm{~K}$ and $550 \mathrm{~K}$. With increasing temperature the amplitude decreases in a continuous but nonlinear manner. This change was emphasized through a linear fit through the temperature points above $400 \mathrm{~K}$. Such a linear fit has no theoretical basis and is merely a guide to the eye. However, there is obviously a change in the slope around $T \approx 400 \mathrm{~K}$. Because we are probing structural relaxations at nearest neighbor distances that change in dynamics is evidence for a change in dynamics. Yet, the amplitude $S\left(Q_{0}, \omega=0\right)$

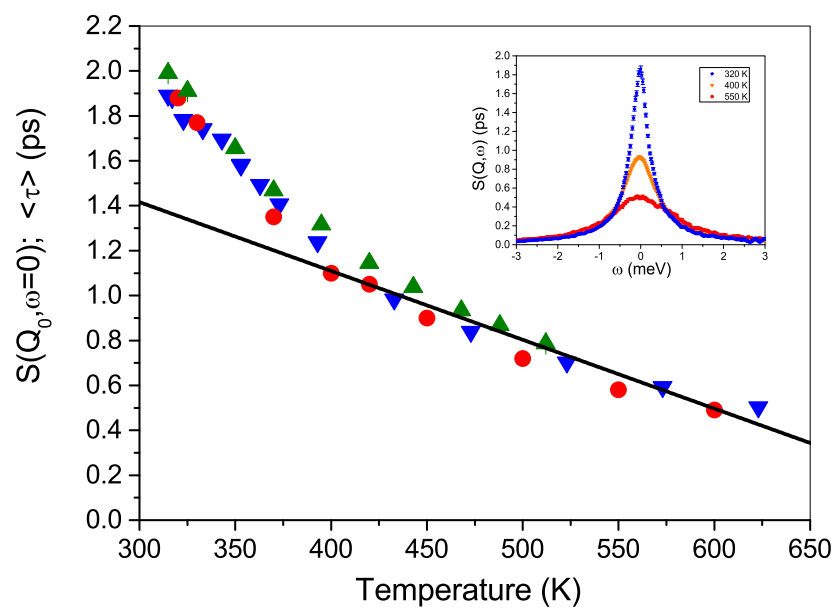

Figure 2. The amplitude $S\left(Q_{0}, \omega=0\right)$ and the average relaxation time $\langle\tau\rangle$ is presented. The different symbols relate to the different experiments: triangle down (FRM), triangle up (IN3, ILL) and circles (IRIS, ISIS Facility). The line is a fit to the high temperature values and represents a mere guide to the eye.

can be considered in a different way. $S\left(Q_{0}, \omega=0\right)$ is related to the intermediate scattering function $F(Q, t)$ through a Fourier transformation in the following way:

$$
S\left(Q_{0}, \omega=0\right)=\left.\int F(Q, t) \exp (i \omega t) d t\right|_{\omega=0}=\int F(Q, t) d t
$$

and the last integral can be regarded as a definition for an average relaxation time:

$$
\langle\tau\rangle=\int F(Q, t) d t
$$

This average relaxation time $\langle\tau\rangle$ is identical to our measured amplitude $S\left(Q_{0}, \omega=0\right)$ and can be interpreted as a decay time of density fluctuations on next neighbor distances. That approach delivers only an average relaxation time and no details about the actual decay of the intermediate scattering function. It might be interpreted as the average time a particle is associated with its nearest neighbors. The changing slope of $\langle\tau\rangle$ with decreasing temperature could then be understood that the stronger increase of the relaxation time is caused by a further relaxation process which sets in at around 400-450 K on cooling. Hence, the change in the average relaxation time at next nearest neighbor distances indicates a change in underlying dynamics in the temperature range 400-450 K.

Near the melting point an additional slow relaxation process has been demonstrated by MD-simulation and explained through mode-coupling theory [28]. That slow process was 
brought into connection with structural freezing and was predicted to increase strongly in the supercooled state. Sophisticated neutron scattering experiments proved the existence of this slow relaxation process near the melting point [10] and a later investigation showed that this slow process disappears at about $400 \mathrm{~K}$ [12], in agreement with the changing slope of the average relaxation time. To directly demonstrate a further relaxation process within an average relaxation time $\langle\tau\rangle$ would need a single precise measurement over a large range of temperatures, which is planned for the near future.

Furthermore, the amplitude $S(Q, \omega=0)$ is connected to a generalized longitudinal viscosity coefficient $\eta_{l}(Q)$. The longitudinal viscosity is a measure for the diffusion of momentum parallel to the velocity of the particles and can be regarded as being sensitive to long lasting atomic-level stress correlations, in our case, on next neighbor distances. The evaluation of $\eta_{l}(Q)$ showed a step-like increase of the generalised viscosity with decreasing temperature at around $400 \mathrm{~K}$ [9]. In addition, MD-simulations of generalized viscosities at the structure factor maximum demonstrated a similar increase with decreasing temperature [29]. The increase in generalized viscosity and the increase of the amplitude $S\left(Q_{0}, \omega=0\right)$, related to the set-in of an additional slow relaxation process, are evidence for a change in the dynamics to a more viscous, solid-like state upon cooling at around 400-450 K .

If the dynamics changes to a more solid-like behavior this should be mirrored in changes of elastic moduli, like the high frequency bulk or shear modulus. Near the melting point the dynamics has been related to high frequency elastic moduli of liquid rubidium [30]. Previously we analysed the inelastic collective dynamics of liquid rubidium in detail and derived from spectra of the current correlation functions dispersion curves over a wide range of momentum vectors [11]. From these dispersion curves phase velocities $c(Q)=\omega / Q$ can be calculated for the acoustic-type excitations. At high frequencies the instantaneous response of the system is probed and a high frequency phase velocity can be defined: $c_{\infty}(Q)=\frac{\omega_{l}}{Q}$, where $\omega_{l}$ is related to the fourth frequency moment and is directly linked to the interaction potential.

This high frequency sound velocity has been related to high frequency elastic moduli [26]:

$$
c_{\infty}(Q)=\sqrt{\frac{1}{n M}\left(\frac{4}{3} G_{\infty}(Q)+K_{\infty}(Q)\right)}
$$

with $\mathrm{n}$ the particle density, $\mathrm{M}$ the mass of the particle, $G_{\infty}$ the high frequency shear modulus and $K_{\infty}$ the high frequency bulk modulus. Due to the isotropy of the system and in the low $\mathrm{Q}$ limit this equation can be written: $c_{\infty}(Q)=\sqrt{\frac{3 G_{\infty}(Q)}{n M}}$ and hence a high frequency shear modulus $G_{\infty}$ can be derived from the measured data. The $c_{\infty}(Q)$ values from the measurement [11] have been averaged between $Q=0.45-0.55 \AA^{-1}$ and then $G_{\infty}$ has been calculated accordingly. Fig 3 shows the obtained shear modulus $G_{\infty}$ with rising temperature. The value for solid rubidium was derived from ultrasound velocity measurements using the longitudinal sound velocity [31]. A small change occurs from the solid state to the liquid state near the melting point. There are only four data points in the liquid state, however, a change in the temperature dependence of the shear modulus is evident for the higher two temperature points. Apparently, above $420 \mathrm{~K}$ the rigidness of liquid rubidium decreases with a higher rate than at lower temperature. That softening of the generalised elastic modulus agrees with a reduction in generalised viscosity in this temperature range, although both experiments are monitoring different spatial correlations [9]. 


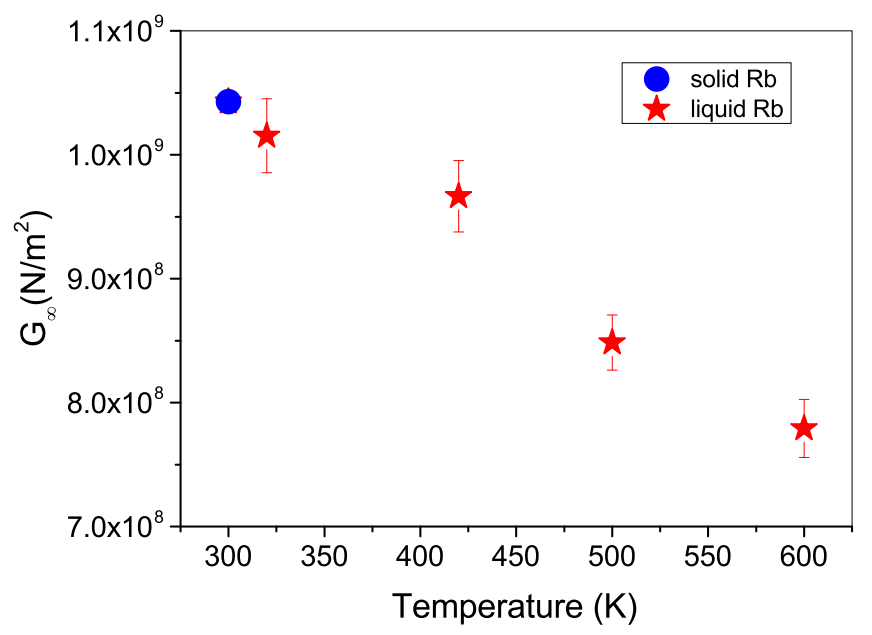

Figure 3. The derived high frequency shear modulus $G_{\infty}$ for the liquid and the solid state is plotted.

\section{Conclusion}

For liquid rubidium the thermal expansion coefficient changes its temperature dependence between $400 \mathrm{~K}$ and $500 \mathrm{~K}$ significantly. On a microscopic scale the amplitude $S\left(Q_{0}, \omega=0\right)$, related to dynamics on next-neighbor distances, changes its slope in the same temperature range. The average relaxation time of density fluctuations at this momentum transfer follows the same trend. An explanation for this change in dynamics might be the set-in of an additional slow relaxation process upon cooling, which has been related to structural freezing. These changes signal a transition between different dynamical regimes in liquid rubidium. Furthermore, in the same temperature range a decrease of generalised viscosity and a softening of the high-frequency shear modulus was observed upon heating. These evidences can be summarized that liquid rubidium shows two different dynamical regimes, a low temperature viscous one and a high temperature fluid one. A similar behavior in the amplitude and generalised viscosity was observed in liquid lead and aluminium [32, 33]. Whether such a transition from a more solid-like to a fluid-like response is a universal behavior in liquid metals needs further investigations.

\section{References}

[1] J.R.D. Copley and S. W. Lovesey Rep. Prog. Phys. 38461 (1975)

[2] T. Scopigno, G. Ruocco and F. Sette Rev. Mod. Phys. 77881 (2005)

[3] J.R.D. Copley and J.M. Rowe Phys. Rev. Lett. 3249 (1974)

[4] T. Bodensteiner, C. Morkel, W. Gläser and B. Dorner Phys. Rev. A 455709 (1992)

[5] H. Sinn, F. Sette, U. Bergmann, C. Halcoussis, M. Krisch, R. Verbeni and E. Burkel Phys. Rev. Lett. 781715 (1997)

[6] W.C. Pilgrim, S. Hosokawa, H. Saggau, H. Sinn and E. Burkel J. Non-Cryst. Solids 25025296 (1999)

[7] L.E. Bove, B. Dorner, C. Petrillo, F. Sacchetti and J.B. Suck Phys. Rev. B 68024208 (2003) 
[8] W.-C. Pilgrim and C. Morkel, J. Phys.: Condens Matter 18 R585 (2006)

[9] F. Demmel, A. Diepold, H. Aschauer and C. Morkel, Phys. Rev. B 73104207 (2006)

[10] F. Demmel, P. Fouquet, W. Häussler and C. Morkel, Phys. Rev. E 73032202 (2006)

[11] F. Demmel, D. Pasqualini and C. Morkel, Phys. Rev. B 74184207 (2006); F. Demmel, A. Diepold, H. Aschauer and C. Morkel, J. Non-Cryst. Solids 3533164 (2007)

[12] F. Demmel and C. Morkel, Phys. Rev. E 85051204 (2012)

[13] A. Rahman, Phys Rev. Lett. 3252 (1974)

[14] G. Kahl and S. Kambayashi, J.Phys.: Condens. Matter 610897 (1994)

[15] D. Pasqualini, R. Vallauri, F. Demmel, Chr. Morkel and U. Balucani, J Non-Cryst. Solids 250-252 76 (1999)

[16] F. Shimojo and Y. Zempo, Phys. Rev. B 529320 (1994); S. Munejiri, F. Shimojo and K. Hoshino, J. Phys.: Condens Matter 124313 (2000)

[17] J.F. Wax, R. Albaki and J.L. Bretonnet, Phys. Rev. B 65014301 (2001)

[18] P. Chieux, J. Dupuy-Philon, J. Jal and J.B. Suck, J. Non-Cryst. Solids 205-207, 370 (1996); G. Pratesi, J.B. Suck and P.A. Egelstaff, J. Non-Cryst. Solids 250-252 91 (1999)

[19] K. Matsuda, K. Tamura and M. Inui, Phys. Rev. Lett. 98, 096401 (2007)

[20] R. Winter, C. Pilgrim, F. Hensel, C. Morkel and W. Gläser, J Non-Cryst. Solids 156-158 9-14 (1993)

[21] W.C. Pilgrim, M. Ross, L.H. Yang and F. Hensel, Phys. Rev. Lett. 783685 (1997)

[22] L. Köster, K. Knopf and W. Waschkowski, Z. Phys A 301 215-226 (1981)

[23] F. Demmel, A. Fleischmann and W. Gläser, Nucl. Instr. Meth. A 416 115-122 (1998)

[24] W. Ohse Handbook of thermodynamic and transport properties of alkali metals (Blackwell scientific Publications Academic Press, Oxford 1985)

[25] L.D. Landau and E.M. Lifshitz Statistical Physics p353 (Pergamon Press, Oxford 1970)

[26] J.P. Boon and S. Yip Molecular Hydrodynamics McGraw Hill (1980)

[27] P.G. deGennes, Physica 25, 825 (1959)

[28] U. Balucani and R. Vallauri, Phys. Rev. A 402796 (1989)

[29] D. Bertolini, F. Demmel and A. Tani, Phys. Rev. B 76094204 (2007)

[30] Chr. Morkel, T. Bodensteiner and H. Gemperlein, Phys. Rev. E 472575 (1993)

[31] W. Schaffs Landolt Börnstein, Neue Serie Band 5, p236, Springer, Berlin-Heidelberg, (1967)

[32] F. Demmel, W. S. Howells and C. Morkel, J.Phys.: Condens. Matter 20205106 (2008);

F. Demmel, W. S. Howells, C. Morkel and W.C. Pilgrim, Z Phys Chem 22483 (2010)

[33] F. Demmel, A. Fraile, D. Szubrin, W.C. Pilgrim and C. Morkel, J.Phys.: Condens. Matter 27455102 (2015) 